\title{
Initial Experimental Characterization of a New Ultra-High Resolution FTICR Cell with Dynamic Harmonization
}

\author{
Eugene N. Nikolaev, ${ }^{1,2,3}$ Ivan A. Boldin, ${ }^{1,2}$ Roland Jertz, ${ }^{4}$ Gökhan Baykut ${ }^{4}$ \\ ${ }^{1}$ The Institute for Energy Problems of Chemical Physics, Russian Academy of Sciences, Leninskij pr. 38, k.2, Moscow, \\ Russia119334 \\ ${ }^{2}$ Institute of Biochemical Physics, Russian Academy of Sciences, Moscow, Russia \\ ${ }^{3}$ The Institute of Biomedical Chemistry, Russian Academy of Medical Sciences, Moscow, Russia \\ ${ }^{4}$ Bruker Daltonik GmbH, Bremen, Germany
}

\begin{abstract}
A new Fourier transform ion cyclotron resonance (FTICR) cell based on completely new principles of formation of the effective electric potential distribution in Penning type traps, Boldin and Nikolaev (Proceedings of the 58th ASMS Conference, 2010), Boldin and Nikolaev (Rapid Commun Mass Spectrom 25:122-126, 2011) is constructed and tested experimentally. Its operation is based on the concept of electric potential space-averaging via charged particle cyclotron motion. Such an averaging process permits an effective electric force distribution in the entire volume of a cylindrical Penning trap to be equal to its distribution in the field created by hyperbolic electrodes in an ideal Penning trap. The excitation and detection electrodes of this new cell are shaped for generating a quadratic dependence on axial coordinates of an averaged (along cyclotron motion orbit) electric potential at any radius of the cyclotron motion. These electrodes together with the trapping segments form a cylindrical surface like in a conventional cylindrical cell. In excitation mode this cell being elongated behaves almost like an open cylindrical cell of the same length. It is more effective in ion motion harmonization at larger cyclotron radii than a Gabrielse et al.-type (Int J Mass Spectrom Ion Processes 88:319-332, 1989) cylindrical cell with four compensation sections. A mass resolving power of more than twenty millions of reserpine $(\mathrm{m} / \mathrm{z} 609)$ and more than one million of highly charged BSA molecular ions $(\mathrm{m} / \mathrm{z} 1357)$ has been obtained in a $7 \mathrm{~T}$ magnetic field.
\end{abstract}

Key words: FT ICR MS, Penning trap, Dynamic harmonization, Ultra-high mass resolution

\section{Introduction}

$\mathrm{I}_{\mathrm{s}}^{\mathrm{n}}$ $\mathrm{n}$ order to let an ion cloud generate a long time domain signal in a Fourier transform ion cyclotron resonance (FTICR) mass spectrometer and, hence, to increase its resolving power it is necessary not only to have an ultra high vacuum but also to keep the cyclotron frequency and phase the same for all ions of the same mass-to-charge ratio during the signal detection time. In general, even in a perfect homogenous magnetic field, this is not the case due to the

Correspondence to: Eugene N. Nikolaev; e-mail: ennikolaev@rambler.ru necessity of an electric field, which is required for trapping the ions in axial direction. In conventional ICR cells, such as cubic, cylindrical, or "open cells" ions of different zoscillation (trapping-oscillation) amplitude have slightly different so-called "effective" cyclotron frequencies. Generally speaking, the effective cyclotron frequency is equal to the cyclotron frequency (the frequency of rotation in magnetic field in absence of any electric field) minus the drift motion frequency (the frequency of motion perpendicular to both magnetic and electric fields), which converges into magnetron motion for hyperbolic geometry electric field distribution. The effective cyclotron frequency is lower for 
higher z-oscillation amplitude because the drift frequency is linearly proportional to the electric potential gradient perpendicular to magnetic field. This gradient increases with increasing z-amplitude in cubic and cylindrical cells. It results in gradual dephasing of an ion packet (due to high z-amplitude ions rotating with smaller circular velocities) and, consequently, in signal decay. This effect limits the signal duration, causes a frequency drift, and, hence, reduces the resolving power of an FTICR mass spectrometer. Signal duration increases in higher magnetic fields because the drift motion frequency is inversely proportional to magnetic field. Signal duration does also significantly increase when the number of ions in a cell is sufficient for phase locking [4]. The effect of phase locking may prolong the signal detection time but, in turn, it decreases the resolving power because ions of close mass-to-charge ratios get phase locked, i.e., not resolved in FTICR spectrum.

There are two approaches to reduce electric field influences. The first one is to reduce a radial electric field in the region of ion detection [5-8]. The second one is to create an electric field configuration that leaves the cyclotron frequency of ions independent of their axial motion - that is a hyperbolic field:

$$
\Phi(r, z)=\frac{1}{2} \gamma\left(2 z^{2}-r^{2}\right),
$$

where $\Phi$ is the electric potential, $r=\sqrt{x^{2}+y^{2}}, \gamma$ is a coefficient proportional to the trapping voltage. The $\mathrm{z}$-axis is directed along the magnetic field vector. For this potential distribution, assuming the magnetic field to be uniform, the equations of motion can be solved exactly [9]. In this field, the variables get separated and the motion of an ion consists of three independent modes:

1) Oscillation along z-axis at $\omega_{z}$ frequency that is defined by

$$
\omega_{z}=\sqrt{\frac{2 q \gamma}{m}}
$$

2) Rotation in xy-plane at $\omega_{+}$frequency, i.e., cyclotron motion,

$$
\omega_{+}=\frac{\omega_{c}}{2}+\sqrt{\left(\frac{\omega_{c}}{2}\right)^{2}-\frac{\omega_{z}^{2}}{2}}
$$

3) Rotation in xy-plane at $\omega_{-}$frequency, i.e., magnetron motion,

$$
\omega_{-}=\frac{\omega_{c}}{2}-\sqrt{\left(\frac{\omega_{c}}{2}\right)^{2}-\frac{\omega_{z}^{2}}{2}} ;
$$

Where $\omega_{c}=q B / m, q$ and $m$ are particle's charge and mass, $B$ is magnetic field strength [9].
One can see that the $\omega_{+}$frequency (effective cyclotron frequency for this case as we called it earlier), which is detected during a FTICR experiment, stays the same for all ions of the same mass-to-charge ratio that are present in an ICR cell, being independent on ions' initial positions and velocities.

A nearly ideal hyperbolic field is provided by a 3D hyperbolic trap (see for instance [10,11]), but it has the major disadvantage of not effective usage of room temperature bore space of high magnetic field homogeneity, i.e., the region where ions can circle is much smaller than the magnet bore. Because of space charge effects such cells will have limited charge capacity, hence limited dynamic range. Another approach to create an ICR cell with trapping field that is close to hyperbolic (also called "harmonization" of an ICR cell [1]) is the segmentation of electrodes. Gabrielse et al. implemented this approach by inserting compensation rings into an open cylindrical cell [3] between the center section and the trapping sections so that it became a five-section compensated cell. This idea was recently pursued in FTICR mass spectrometry by Tolmachev et al. [12] and Gross and coworkers [13]. The compensated cell described in reference [12] is a seven-section cell having two compensation rings on each side of the center section, whereas the one by Gross et al. [13] is a nine-section cell with three compensation rings on each side of the center section. Other segmentation methods involve a matrix cell by Marshall's group [14] and segmented cells (based on cell segmentation in axial direction) developed by Bruce et al. [15], Barlow and Tinkle [16] at Pacific Northwest National Laboratory (PNNL). All these harmonization methods provide hyperbolic electric fields only in a region around the center of the cell. The size of this region depends on the deviation of the harmonized field from the ideal hyperbolic field needed to remove dephasing during detection time. So it depends on the resolving power we aim for. Deviations from harmonicity show up at high excitation radii, when ions get closer to the electrodes, and at high zamplitudes. The method described in the references [1,2] extends the region of harmonicity potentially to the entire cell volume. It is based on subdividing cell cylindrical surface into segments with shapes producing quadratic dependence on axial coordinate of an averaged (along cyclotron orbit) electric potential at any radius of cyclotron motion. Due to the presence of a high magnetic field ions rotate in xy-plane at a frequency that is much higher than the z-oscillation frequency and drift frequency. In this new approach to trapping field harmonization in ICR cells, instead of attempting to create a truly harmonic field in the cell, we create a potential distribution that would be harmonic upon being averaged over one cyclotron period.

\section{Experimental}

\section{The Cell}

The performance of this new type of ICR cell was analyzed theoretically, both analytically and by digital simulations $[1,2]$, 
using an ion motion simulation program [14] with ion-ion and ion-image charge interactions turned off. Cell design and electrode geometry were chosen based on these simulations. The picture of the cell is presented in Figure 1a. The length $L$ of the cell is $150 \mathrm{~mm}$, the inner diameter is $56 \mathrm{~mm}$. The curved slits are $1 \mathrm{~mm}$ wide, defined by equation

$$
\alpha=\frac{2 \pi}{N} n \pm \alpha_{0}\left(1-\left(\frac{2 z}{L}\right)^{2}\right) ; n=0,1, \ldots,(N-1)
$$

where $\alpha$ is the azimuthal angle, $N=8, \alpha_{0}=\pi / 8-\pi / 60$. Ideally, $\alpha_{0}$ should be equal to $\pi / 8$ so that adjacent slits are tangential to the mid-plane of the cell. But this is quite inconvenient for manufacturing therefore we leave "straits" of approximately $2 \mathrm{~mm}$ thickness. The inner surface of the end-cap electrodes was made spherical as this was easier to manufacture than a hyperbolic. A spherical surface differs from the hyperbolical one by $0.02 \mathrm{~mm}$ at maximum, what we consider insignificant. The end-cap electrodes have orifices of $6 \mathrm{~mm}$ diameter in their centers. An additional electrode at the entrance of the cell is added here to provide focusing of incoming ions.

On both end cap electrodes as well as the entrance lens a typical DC voltage of $1.5 \mathrm{~V}$ is directly applied during the detection sequence, the same DC voltage is applied to the convex electrodes of the cylindrical surface, while the concave electrodes remain grounded. The potential distribution during the detection period at longitudinal section and two cross-sections is shown in Figure 1b. During the injection of ions the entrance lens and the front end cap electrode are pulsed down to typically $-10 \mathrm{~V}$. The electric
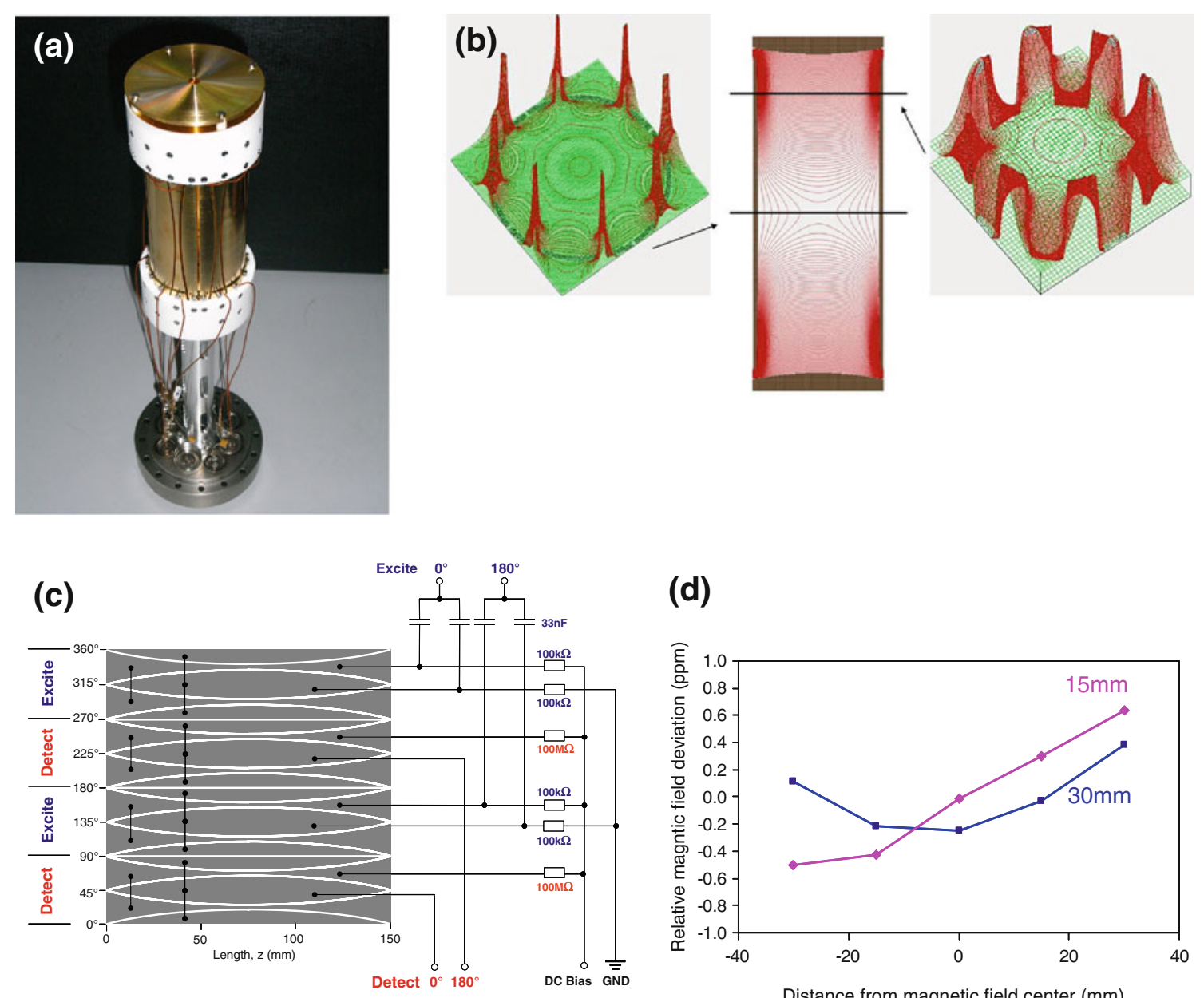

(d)

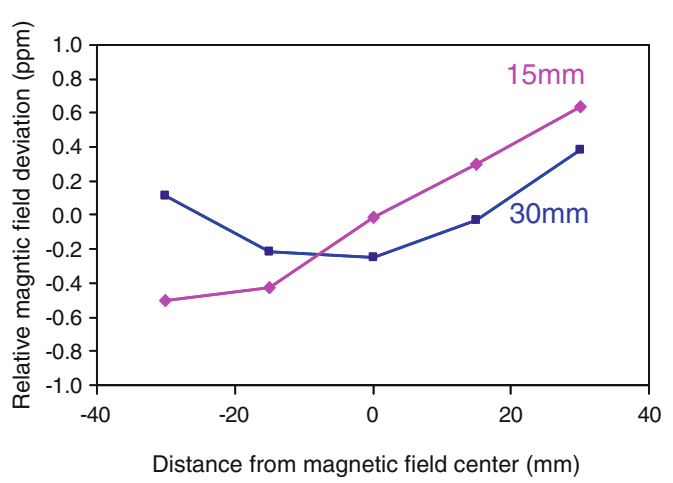

Figure 1. (a) Picture of the new ICR cell. (b) Potential distribution at longitudinal section of the cell and two cross-sections during detection. (c) Electric connections of the cylinder surface electrodes in two-dimensional representation. All concaveshaped electrodes are connected to the trapping voltage; the convex-shaped electrodes are grounded. The complete electrode set is divided into four sections each forming a $90^{\circ}$ angle. The two excitation sections consist of one full and two half convex electrode segments plus two concave electrodes. The excitation chirp is applied to all electrodes of the detection section via a $33 \mathrm{nF} / 100 \mathrm{kOhm}$ high pass filter. The two opposite detection sections are identically structured as the excitation sections. The preamplifier is connected to the convex electrode and the two half convex segments. The concave electrodes are not used for detection, the trapping voltage is applied via 100 MOhm resistors, see text. (d) Relative magnetic field values, averaged over radial planes as a function of magnetic field axis position. The magnetic field was measured on two different cylinder surfaces of 1.5 and $3 \mathrm{~cm}$, respectively. The connecting lines are plotted to guide the eye 
connections to the electrodes at the cylindrical surface are shown in Figure 1c. The connection of the excitation sections are realized using four RC (resistance capacitance) high pass filter circuits, since both the concave-shaped DC biased trapping and the convex-shaped grounded electrodes need to be decoupled from the dipolar radio frequency (rf) excitation voltage. The pre-amplifier is only connected to the ground electrodes of the detection sections, since the DC bias voltage would introduce an additional noise of two orders of magnitude. In order to minimize the noise induced into the detection electronics even further also the DC biased trapping electrodes of the detection sections are connected via resistors. The complete electric circuit was optimized to generate minimum electric noise picked up in the detection electronics using the signal circuit simulation tool LTspice IV, developed by Linear Technology (freeware).

Experiments were performed using a special ESI-Qh-FTICR mass spectrometer. An actively shielded 7 Tesla superconducting magnet (Bruker Biospin, Wissembourg, France) with a room temperature bore of $160 \mathrm{~mm}$ diameter was used. The mass spectrometer was equipped with an electrospray ion source, a quadrupole mass selector, a hexapole collision cell, and a hexapole ion guide for transferring ions to the ICR cell. Ions were generated in the electrospray ion source that contains a dual ion funnel system and a hexapole ion guide. Passing through the glass electrospray capillary with metallized ends ions entered into the first vacuum stage orthogonal to the ion funnels, i.e.; orthogonal to the transfer axis of the FTICR system. On their travel to the ICR cell, ions were accumulated in the collision cell. They were then extracted and transferred through the hexapole ion guide into the ICR cell in the center of the magnetic field. The magnetic field in the ICR cell region was also measured directly using an NMR probe. The homogeneity of the magnetic field is characterized by the maximum difference of two magnetic field values measured on a surface of two cylinders around the magnetic field center with $1.5 \mathrm{~cm}$ and $3 \mathrm{~cm}$ radii, and $6 \mathrm{~cm}$ length. In this particular magnet, $1.52 \mathrm{ppm}$ and $1.96 \mathrm{ppm}$ were determined, respectively. By averaging the field values in the radial plane for each measured axial position, a linear shift from $-0.5 \mathrm{ppm}$ to $+0.6 \mathrm{ppm}$ around the magnetic field center was found on the $1.5 \mathrm{~cm}$ radius cylinder and a second order shift from $-0.2 \mathrm{ppm}$ to $+0.2 / 0.4 \mathrm{ppm}$ on the $3 \mathrm{~cm}$ radius cylinder (Figure $1 \mathrm{~d}$ ).

\section{Materials Used}

The new ICR cell was experimentally tested for maximum achievable mass resolving power, using reserpine (\#R-0875 by Sigma Aldrich, Munich, Germany) for single isotope mass detection and bovine serum albumin (BSA) (no. 1062 by Gerbu Biochemicals, Gaiberg, Germany) for resolving multiplets of highly charged isotopes. Reserpine was sprayed by direct infusion in a $10 \mathrm{fmol} / \mu \mathrm{L}$ solution in water/methanol (1:1 in vol) into which $0.1 \%$ formic acid was added. Nitrogen was used as drying gas. The temperature of the drying gas heater was set to $200{ }^{\circ} \mathrm{C}$. The continuously generated singly protonated reserpine ions $(\mathrm{m} / \mathrm{z} 609)$ were monoisotopic isolated in the quadrupole mass selector and subsequently accumulated in the collision cell for typically $0.5 \mathrm{~s}$ The captured ions were excited by a dipolar broadband excitation chirp (from 150 to $3500 \mathrm{Da}$ ) applied for about $16 \mathrm{~ms}$, with each single frequency applied for $15 \mu \mathrm{s}$. The detection was done in the heterodyne mode, using up to $512 \mathrm{k}$ data points, where one single scan was acquired. The time domain signal was transformed using the FFT magnitude calculation, including a zero filling to four times of the initial time domain size. No apodization window was applied.

A $3 \mathrm{pmol} / \mu \mathrm{L}$ solution of BSA in water/methanol $(1: 1$ in vol) with $0.1 \%$ formic acid added was sprayed. The BSA was measured in both broadband and in heterodyne detection mode. The high resolution BSA spectra were achieved by isolating one single charge state in the quadrupole mass selector. Data acquisition was performed very similar as described for reserpine except that 100 scans were accumulated in case of both broadband and heterodyne detection mode.

\section{Results}

\section{High Mass Resolving Power}

In the case of the single isotope detection of reserpine, transient lengths of up to $3 \mathrm{~min}$ could be achieved, see Figure $2 b$. This results in a mass resolving power of

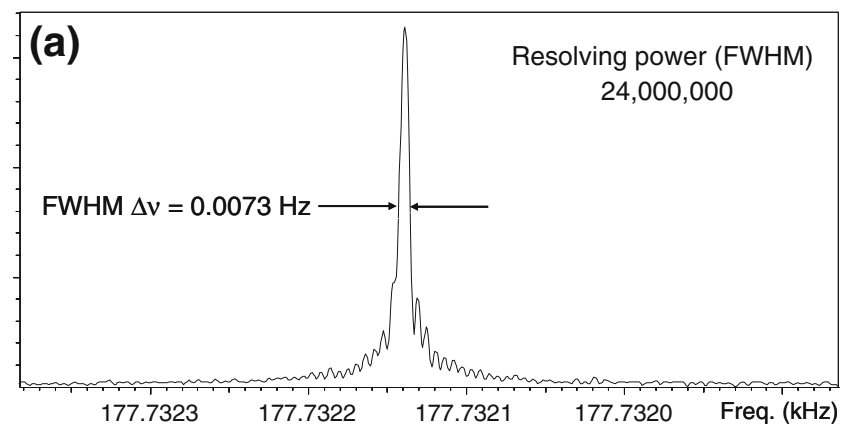

(b)

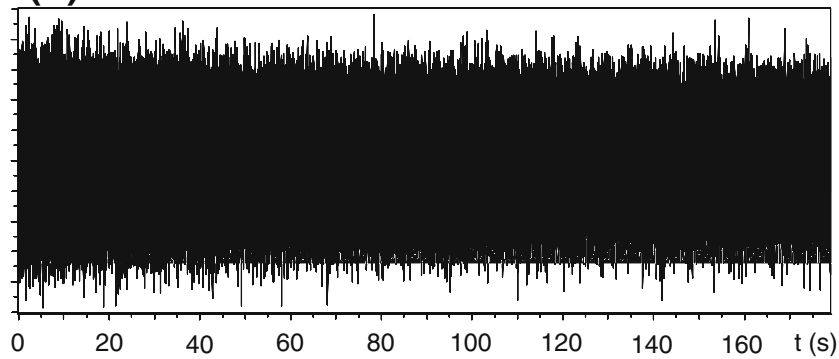

Figure 2. (a) Frequency spectrum (not calibrated) of the mono isotopic peak of singly charged, protonated reserpine $(\mathrm{m} / \mathrm{z}$ 609.28066) with a resolving power of $24,000,000$, resulting from magnitude FFT calculation without apodization. (b) Time domain spectrum of reserpine, detected over $3 \mathrm{~min}$ 
$24,000,000$ in the magnetic of 7 Tesla. A peak-to-peak rf excitation voltage of $20 \mathrm{~V}$ was applied.

The theoretical resolving power at zero pressure limit can be calculated using the following numerical-value equation [9]:

$$
\frac{v}{\Delta v_{50 \%}}=\frac{m}{\Delta m_{50 \%}}=\frac{1.274 \times 10^{7} z B T_{a c q}}{m}
$$

with $\mathrm{z}$ the charge state, $\mathrm{B}$ the magnetic flux density in Tesla, $\mathrm{T}_{\mathrm{acq}}$ the acquisition time in seconds, $v$ the frequency, $\Delta v_{50 \%}$ the width (FWHM) of the resonance peak, $\mathrm{m}$ the mass, and $\Delta \mathrm{m}_{50 \%}$ the width (FWHM) of the mass spectral peak in atomic mass units. Inserting $\mathrm{T}_{\mathrm{acq}}=180 \mathrm{~s}$ for the acquired three-minute transient, $\mathrm{m} / \mathrm{z} 609$ for the protonated reserpine molecule, the theoretical resolving power is calculated to be $26,358,620$. Hence, with the experimentally achieved transient length of $180 \mathrm{~s}$ we are still within the zero-pressure limit.

It can be easily seen from the reserpine peak shape in the spectrum, shown in Figure 2a, that the transient evolves over the complete detection time, while undergoing a slight decay (see Figure 2b). Except a small shoulder on the left, it shows the typical shape of a pure sine wave folded by a square function, the finite detection time, resulting in the typical sidebands. The plotted resonance peak was not apodized.

The multiplet detection measurements of BSA are shown in Figure 3. A typical broad band mass spectrum is shown in Figure $3 \mathrm{a}$, where several charge states ranging from $37+$ up to $54+$ protonated BSA ions were detected at very low resolution, just showing the envelopes of the charge states. The high resolution measurements were performed in the heterodyne detection mode. Unlike in the Reserpine measurements frequency time step duration of the excitation chirp of $5 \mu \mathrm{s}$ and a peak-to-peak excitation voltage of $145 \mathrm{~V}_{p p}$ was applied here.

Figure $3 \mathrm{~b}$ shows a $22 \mathrm{~s}$ long transient after selecting the charge state group of $49+(\mathrm{m} / \mathrm{z}$ 1357) in the quadrupole mass selector. The typical "beats" generated by a mixture of very narrow lying isotopes clearly prove the transient decay over the detection time. For this spectrum 100 single transients were accumulated, since the signal intensity (ion population in the cell) had to be kept low in order to avoid peak coalescence. The subsequent magnitude FFT calculation results in the mass spectrum, shown in Figure $3 \mathrm{c}$ and $\mathrm{d}$ demonstrating a clear isotope resolution of the isolated charge state and a mass resolving power of 1,200,000. According to Equation 8 from the Appendix, excitation parameters correspond in both reserpine and BSA cases to a cyclotron radius of $15 \%-20 \%$ of the cell radius. We should note that we have reasonably high resolution at larger radii but the maximum resolving power is observed at relatively small radii. This behavior might be caused by incomplete averaging of the field by cyclotron motion at larger radii with this relatively small number of segments. The other possible reason for lower resolving power at large cyclotron radii is the magnetic field inhomogeneity. As described above, the magnetic field homogeneity was measured to be $1.96 \mathrm{ppm}$ as determined by a measurement on a surface of a cylinder of $3 \mathrm{~cm}$ radius and a length of $6 \mathrm{~cm}$ in the magnetic field center.

\section{Comparison with a Compensated Cell}

The new ICR cell has already been compared theoretically $[1,2]$ to one of the latest developments in ICR cell harmonization - to the compensated seven-section cell [12]. Computational experiments have shown that for the case of Gabrielse type cell the dephasing rate is 3.5 times higher compared with the new cell resulting in the same decrease in mass resolving power. An experimental comparison was made using a compensated cell which was built in our labs according to the dimensions of the compensated sevensection cell [12]. The optimum compensation electrode voltage settings for the measurements evaluated (in broadband detection mode) as shown in Figure 5 have been found to be the same as described in reference [12]. In the case of ultra high resolution measurements both sets of compensation voltages had to be adjusted slightly different towards lower voltages in order to obtain the maximum resolving power.

Figure 4 shows the comparison of the progression of the measured cyclotron frequency during the same experiments in the compensated cell and in the new ICR cell as a function of the ion cyclotron radius in percent of the cell radius calculated from 3rd-to-1st harmonics' intensity ratio using Equation 8 from the Appendix. Again, reserpine was used as sample. The number of ions in the measurements can be estimated to be about 500 ions. The ion population had to been kept quite low in order to avoid frequency shifts due to the space charge at low excitation radii. In the new ICR cell a very small but steady and linear cyclotron frequency shift of $1.4 \mathrm{ppm}$ over the complete cell volume is observed. A possible reason for this shift is magnetic field inhomogeneity (Fig. 1d). In the case of the compensated ICR cell, the cyclotron frequency at lower orbits is almost constant, but at an excitation to $40 \%$ of the cell radius it starts decreasing and finally drastically drops at $50 \%$. This indicates that in the new ICR cell type the ions are exposed to a much more uniform electric field over the complete cell area as predicted by theory [2].

\section{Discussion}

In the compensated ICR cells the harmonization is done by tuning/optimizing the different voltages applied to the compensation sections. In the recent literature two basic compensated cells are described, the referred seven-section cell [12] and the referred nine-section cell [13]. These cells have four and six compensation ring sections, respectively, in order to apply various compensation voltages. In those compensated cells the process of electrode voltage tuning to 

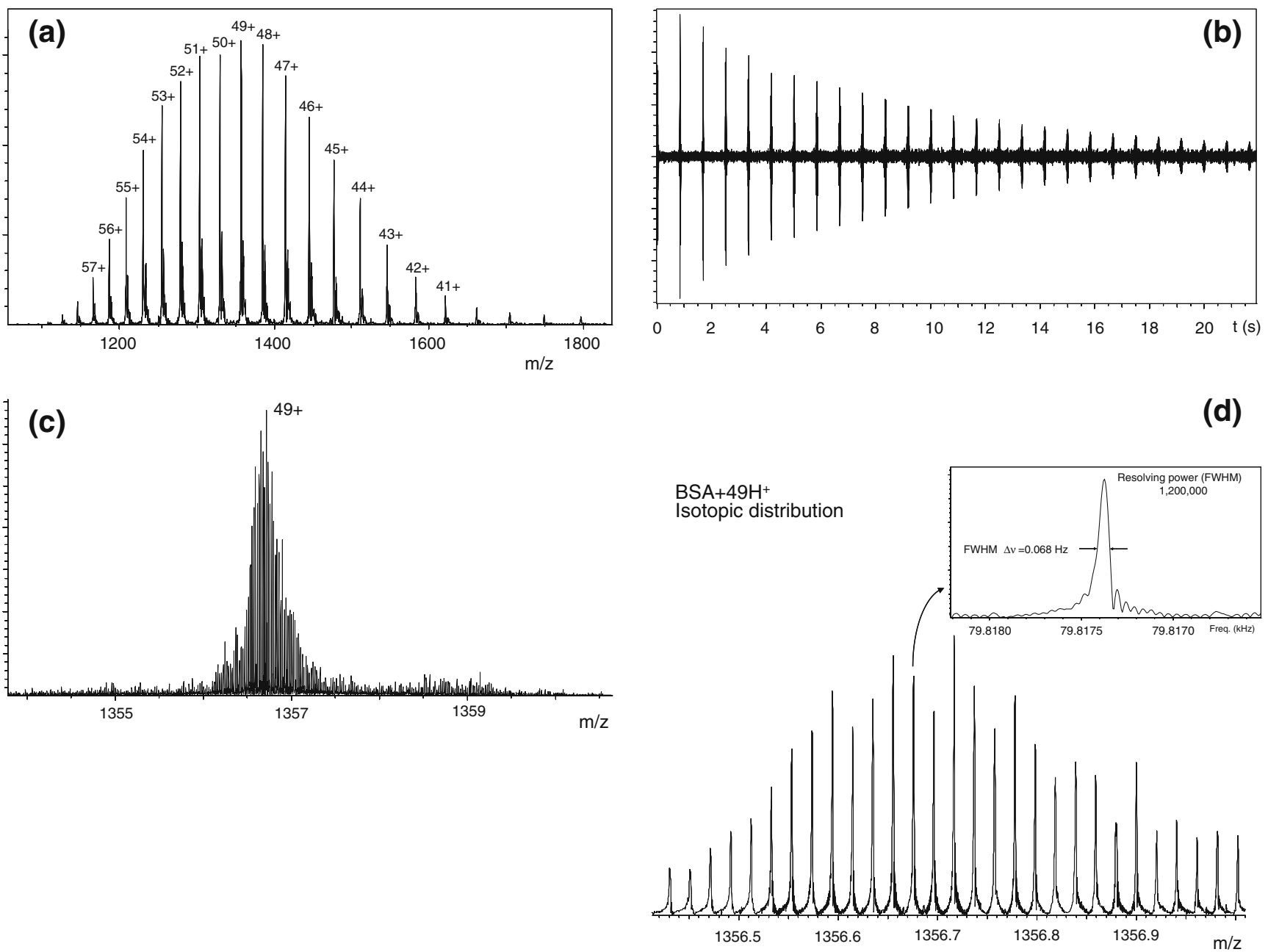

Figure 3. (a) Broadband mass spectrum of bovine serum albumin (BSA) showing the distribution of charge states ranging from $37+$ up to 60+. (b) Time domain spectrum of the isolated charge state 49+ (BSA with 49 protons) detected over $22 \mathrm{~s}$. (c) Mass spectrum of the isolated charge state isotopic distribution of $49+$ of BSA achieved by magnitude FFT calculation from the transient (b). (d) Zoom in of the mass spectrum (c), demonstrating a resolving power of 1,200,000

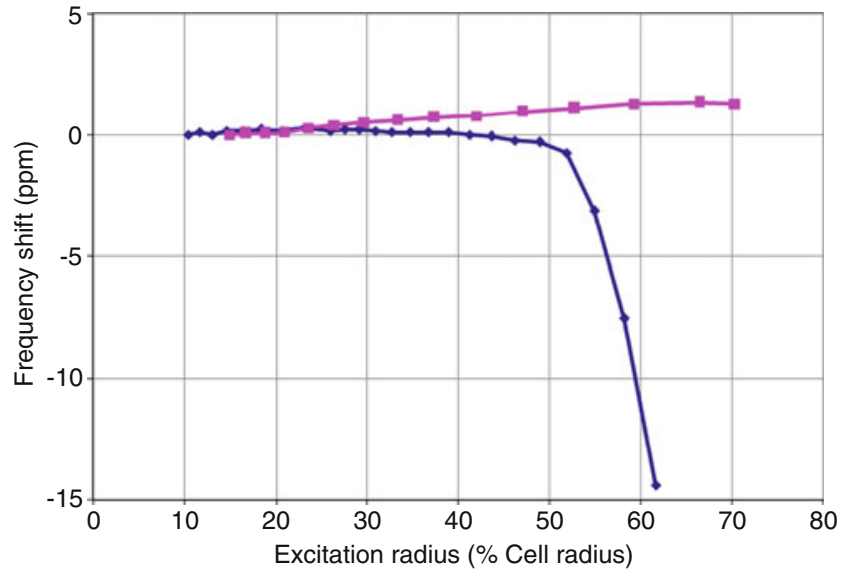

Figure 4. Comparison of the new cell (upper curve, purple) and a compensated cell (lower curve, blue) of dimensions published by Tolmachev et al. [12]. Frequency progression as a function of ion cyclotron motion radius in units of percent of the cell radius defined as described in Appendix. The first measuring point is defined as reference point optimize the compensation/harmonization is more complicated than in the new ICR cell presented in this paper, where the harmonization is based on the space-averaging of the potential generated by one voltage applied to specifically shaped cell plates.

As a result, in this new ICR cell very long transients can be easily obtained and ultra high mass resolving power more than three times higher as in a compensated cell (e.g., the seven-section cell [12]) can be achieved.

Initial magnetron orbit sizes tend to increase continuously with increasing detection time due to collisions with the residual gas. Thus, very low cyclotron excitation amplitudes should rather be chosen in order to acquire extremely long transients. The longer the detection time (transient), the lower should be the excitation amplitude. On the other hand, in order to avoid peak coalescence phenomena and minimize space charge shifts, an excitation of ions to large cyclotron orbits is required, due to the fact that after the excitation the ions occupy a larger volume and their number density drops 
correspondingly. These contradicting phenomena require a careful optimization of the excitation amplitude when performing high resolution experiments for different ions of different compounds.

\section{Perspectives}

The new cell will help increase the maximum mass for which isotopic resolution could be achieved beyond $100 \mathrm{kDa}$, and push top-down proteomics towards higher mass regions. The work in this direction is in progress. Further experiments are needed to find the optimum number of the electrodes. The number of segments in the cell is a variable, which can be changed from 2 to any number technologically possible. It is clear that the larger this number is the more effective is the averaging of the potential. This could be important for large $\mathrm{m} / \mathrm{z}$ ions of which the cyclotron frequencies are comparable with the axial motion frequencies.

\section{Conclusions}

The new method of ICR cell harmonization has been proposed and tested experimentally. The new cell demonstrates the highest performance in mass resolving power and excitation range so far achieved in a $7 \mathrm{~T}$ magnetic field.

\section{Acknowledgments}

The authors acknowledge support for this work by the Russian Foundation for Basic Research through grants 10-04-13306, 0904-12130, and 09-04-12225, the Russian Ministry of Education and Science contracts 14.740.11.0755, 16.740.11.0369, Program "Fundamental science for medicine" RAS and Bruker Daltonics.

\section{Appendix}

\section{Determination of Cyclotron Radius}

In order to characterize experimentally the new ICR cell behavior at different cyclotron radii, we linked it with excitation parameters. Two methods are generally used to determine the cyclotron radius. The first one is based on the assumption that the ion signal intensity drop, as a function of the excitation voltage, corresponds to the ion loss due to the contact with the cell walls. Thus, the excitation voltage at which the ions touch the cell walls can be used to normalize this function and determine the absolute radius for every excitation voltage. The second method is based on measurements of the signal intensity distribution for the harmonics of ions with the same $m / z$, which contains information about the cyclotron radius $[17,18]$. We used the second method as the more objective one because it is difficult to distinguish between the two mechanisms of signal drop due to walltouching and due to the synchronization loss caused by inhomogeneities of the electric field near the wall region. To determine the cyclotron radii, singly protonated isolated reserpine ions were exposed to increasing dipolar excitation rf field amplitudes. The mass spectra were measured in the broadband detection mode and evaluated by determining the simultaneously appearing odd harmonic frequencies of the actual ion signal. By increasing the excitation amplitudes, even higher order harmonic frequency peaks of the actual ion signal could be detected. Figure 5a shows the measured intensities of the ion signal (blue line) and its odd harmonic frequencies as a function of the excitation rf amplitude. The abundance of these odd harmonics can be used as a direct measure of the radius of the cyclotron orbit in a FTICR cell [21]. Of course, we should keep in mind that the ion cloud can have a diameter comparable to the cell entrance diaphragm diameter; therefore such a method of cyclotron radius determination is not very accurate. The intensity drop at higher excitation voltages (Figure 5a) is to be interpreted as the loss of ions due to their interaction with the cell electrodes when circling on large cyclotron orbits or due to the loss of synchronization of their cyclotron motion as pointed out above. From the intensity ratio of the odd harmonics signals to the ion signal, shown in Figure 5b, one can evaluate the post-excitation cyclotron radius for each excitation amplitude in approximation of point charges. We are aware that this method of determination of the absolute value of cyclotron radius is not very accurate but it gives us a correct answer for relative values if we compare the cyclotron radii in two different ICR cells like we did in this research.

In order to correlate relative intensities of harmonics to cyclotron radii, we calculated theoretical spectra of harmonics for different cyclotron radii in point charge approximation. The method we used to obtain these theoretical spectra is based on Green's reciprocity theorem [19]. It can be shown [20] that in a system of grounded electrodes the charge $Q$, induced in one of the electrodes by a point charge $q$, placed in free space in the vicinity of the system of electrodes, can be found as:

$$
Q=-q \frac{\Phi_{q}}{\Phi_{0}}
$$

where $\Phi_{q}$ is the potential, created at the point where the charge $q$ is located, if the voltage $\Phi_{0}$ is applied to that particular electrode, and all the other electrodes remain grounded. In our case, it means that in order to find the theoretical transient, we should calculate "detection field" which is the field created by our cell, if $1 \mathrm{~V}$ is applied to one group of detection electrodes and $-1 \mathrm{~V}$ is applied to the opposite group of detection electrodes. Then we record values of this field along a cyclotron orbit (we used simplified orbit - which is the circle laying in $\mathrm{z}=0$ plane with its center coinciding with the center of the cell) which is the theoretical transient [2]. Then we apply Fourier transform to this transient and obtain theoretical spectrum of harmonics. We repeat this procedure for a series of 
(a)

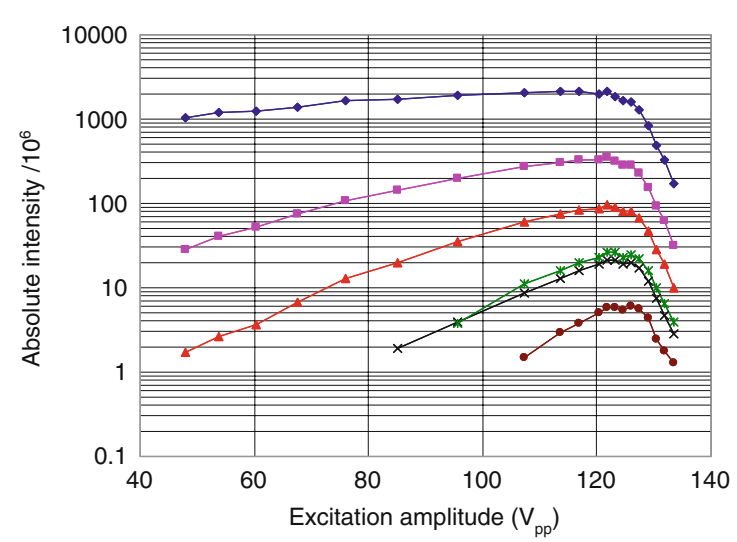

(b)

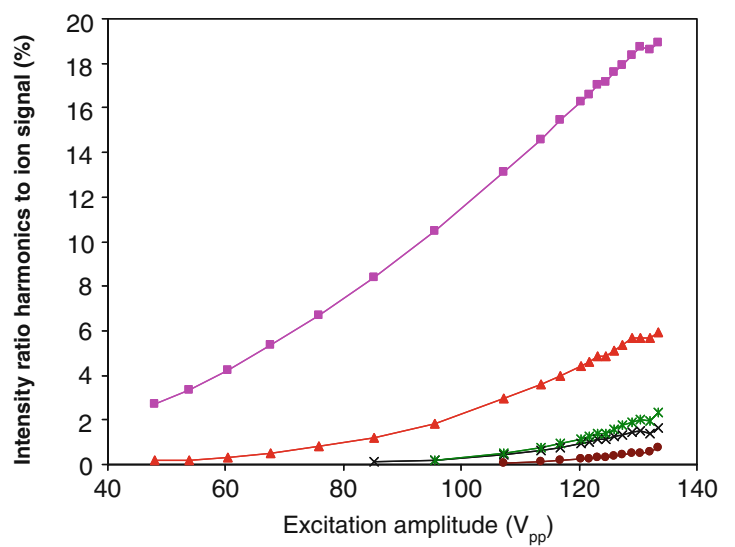

(c)

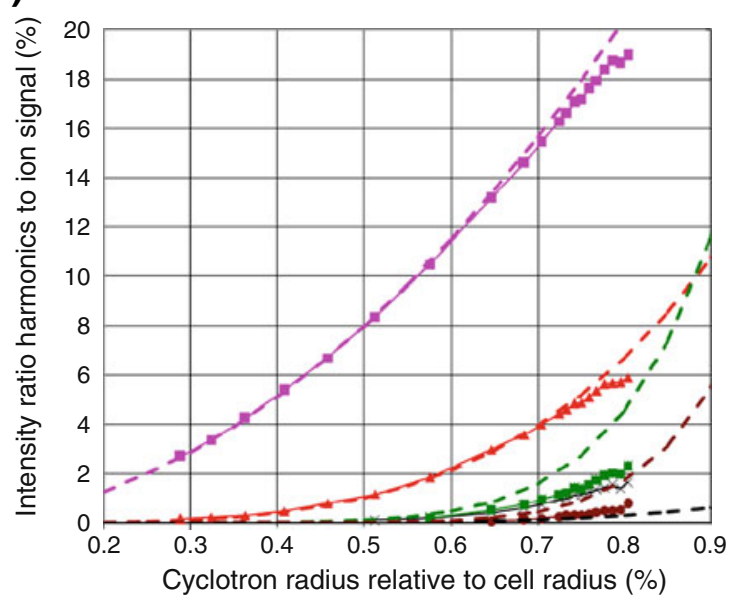

Figure 5. (a) Measured ion signal intensity as a function of the dipolar excitation rf field amplitudes. The blue line indicates the actual ion signal whereas the other lines in purple, red, green, black, and brown show the 3rd, 5th, 7th, 9th, and 11th harmonic frequencies, respectively, all detected simultaneously in the same mass spectrum. (b) Intensity ratios of the uneven harmonic frequencies in units of percent of the referring ion signal as a function of the applied dipolar excitation rf field amplitudes. (c) Theoretically (dashed lines) and experimentally (solid lines) obtained 3rd, 5th, 7th, 9th, and 11th harmonics to ion signal intensity ratios as a function of the cyclotron radius in relation to the cell radius

cyclotron radii to obtain harmonics' intensities dependences on cyclotron radius. We used SIMION 8.0 to calculate the "detection field" and MATLAB 7.5 to interpolate the potential distribution on circles and make Fourier transforms. These dependencies are shown in Figure $5 \mathrm{c}$ as dashed lines. Experimental dependencies (solid lines) are superimposed to give the best match with respect to $3 \mathrm{rd}$ and 5 th harmonics (Figure 5c). Thus, we obtain a linear relationship between excitation amplitude in volts $\left(\mathrm{V}_{p p}\right)$ and cyclotron radius relative to cell radius $(\mathrm{R})$ :

$$
\mathrm{V}_{p p}=166 \times R
$$

(for a dipolar excitation chirp applied for about $16 \mathrm{~ms}$ with a frequency time step duration of $15 \mu \mathrm{s}, \mathrm{V}_{p p}$ in Volts). From Figure 5a we can conclude that we start to see signal degradation at $122 \mathrm{~V}_{p p}$, which corresponds to a cyclotron radius of $73 \%$ of cell radius. We cannot explain this other than by a large ion cloud radius/distribution. Hence, the cyclotron radius determined in this way should be considered as a minimum possible, because we smother the ion signal and suppress high harmonics in case of an ion cloud with real dimensions.

\section{References}

1. Boldin, I. A.; Nikolaev, E.N. Harmonization of Electric Field in FT ICR Cell. The New Approaches. Proceedings of the 58th ASMS Conference on Mass Spectrometry Allied Toppics; Salt Lake City, UT, May, 2010.

2. Boldin, I.A., Nikolaev, E.N.: FTICR cell with dynamic harmonization of the electric field in the whole volume by shaping of excitation and detection electrode assembly. Rapid Commun Mass Spectrom 25, 122$126(2011)$

3. Gabrielse, G., Haarsma, L., Rolston, S.L.: Open-endcap penning traps for high precision experiments. Int J Mass Spectrom Ion Processes 88, 319-332 (1989) 
4. Nikolaev, E.N., Heeren, R.M.A., Popov, A.M., Pozdneev, A.V., Chingin, K.S.: Realistic modeling of ion cloud motion in a fourier transform ion cyclotron resonance cell by use of a particle-in-cell approach. Rapid Commun Mass Spectrom 21, 3527-3546 (2007)

5. Wang, M., Marshall, A.G.: A "screened" electrostatic ion trap for enhanced mass resolution, mass accuracy, reproducibility, and upper mass limit in fourier-transform ion cyclotron resonance mass spectrometry. Anal Chem 61, 1288-1293 (1989)

6. Franzen, J.; Nikolaev, E. 2008. US Patent 7368711.

7. Naito, Y., Fujiwara, M., Inoue, M.: Improvement of the electric field in the cylindrical trapped-ion cell. Int J Mass Spectrom Ion Processes 120 , 179-192 (1992)

8. Weisbrod, C.R., Kaiser, N.K., Skulason, G.E., Bruce, J.E.: Trapping ring electrode cell: A FTICR mass spectrometer cell for improved signal-to-noise and resolving power. Anal Chem 80, 6545-6553 (2008)

9. Marshall, A.G., Hendrickson, C.L., Jackson, G.S.: Fourier transform ion cyclotron resonance mass spectrometry: A primer. Mass Spectrom Rev 17, 1-35 (1998)

10. Van Dyck Jr., R.S., Wineland, D.J., Ekstrom, P.A., Dehmelt, H.G.: High mass resolution with a new variable anharmonicity penning. Trap Appl Phys Lett 28, 446-448 (1987)

11. Rempel, D.L., Ledford Jr., E.B., Huang, S.K., Gross, M.L.: Parametric mode operation of a hyperbolic penning trap for fourier transform mass spectrometry. Anal Chem 59, 2527-2532 (1987)

12. Tolmachev, A.V., Robinson, E.W., Wu, S., Kang, H., Lourette, N.M., Pasa-Tolic, L., Smith, R.D.: Trapped-ion cell with improved DC potential harmonicity for FTICR MS. J Am Soc Mass Spectrom 19, 586-597 (2008)
13. Brustkern, A.M., Rempel, D.L., Gross, M.L.: An electrically compensated trap designed to eighth order for FTICR mass spectrometry. $J \mathrm{Am}$ Soc Mass Spectrom 19, 1281-1285 (2008)

14. Jackson, G.S., White, F.M., Guan, S., Marshall, A.G.: Matrix-shimmed ion cyclotron resonance ion trap simultaneously optimized for excitation, detection, quadrupolar axialization, and trapping. J Am Soc Mass Spectrom 10, 759-769 (1999)

15. Bruce, J.E., Anderson, G.A., Lin, C.Y., Gorshkov, M.V., Rockwood, A. L., Smith, R.D.: A novel high-performance fourier transform ion cyclotron resonance cell for improved biopolymer characterization. $J$ Mass Spectrom 35, 85-94 (2000)

16. Barlow, S.E., Tinkle, M.D.: "Linearizing" an ion cyclotron resonance cell. Rev Sci Instrum 73, 4185-4200 (2002)

17. Nikolaev, E.N., Gorshkov, M.V.: Dynamics of ion motion in an elongated cylindrical cell of an ICR spectrometer and the shape of the signal registered. Int J Mass Spectrom Ion Processes 64, 115-125 (1985)

18. Nikolaev, E.N., Rakov, V.S., Futrell, J.H.: Analysis of harmonics for an elongated FTMS Cell with multiple electrode detection. Int $J$ Mass Spectrom Ion Processes 157/158, 215-232 (1996)

19. Griffith, D.J.: Introduction to electrodynamics, p. 157. Prentice-Hall, New Jersey (1999)

20. Landau, L.D., Lifshitz, E.M.: Electrodynamics of continuous media, p. 7. Pergamon Press, Oxford (1984)

21. Grosshans, P.B., Marshall, A.G.: Cyclotron radius determination in fourier transform ion cyclotron resonance mass spectrometry. Int J Mass Spectrom Ion Processes 115, 1-19 (1992) 